\title{
High expectancy and early response produce optimal effects in sertraline treatment for post-traumatic stress disorder
}

\author{
Belinda Graham, Natalia M. Garcia, Mark S. Burton, Andrew A. Cooper, Peter P. Roy-Byrne, \\ Matig R. Mavissakalian, Norah C. Feeny and Lori A. Zoellner
}

\section{Background}

Better indicators of prognosis are needed to personalise posttraumatic stress disorder (PTSD) treatments.

\section{Aims}

We aimed to evaluate early symptom reduction as a predictor of better outcome and examine predictors of early response.

\section{Method}

Patients with PTSD $(N=134)$ received sertraline or prolonged exposure in a randomised trial. Early response was defined as $20 \%$ PTSD symptom reduction by session two and good end-state functioning defined as non-clinical levels of PTSD, depression and anxiety.

\section{Results}

Early response rates were similar in prolonged exposure and sertraline (40 and $42 \%$ ), but in sertraline only, early responders were four times more likely to achieve good end-state functioning at post-treatment (Number Needed to Treat $=1.8,95 \% \mathrm{Cl}$
1.28-3.00) and final follow-up (Number Needed to Treat $=3.1$, 95\% Cl 1.68-16.71). Better outcome expectations of sertraline also predicted higher likelihood of early response.

\section{Conclusions}

Higher expectancy of sertraline coupled with early response may produce a cascade-like effect for optimal conditions for long-term symptom reduction. Therefore, assessing expectations and providing clear treatment rationales may optimise sertraline effects.

Declaration of interest:

None.

\section{Keywords}

Early response; expectancy; antidepressants; post-traumatic stress disorder.

\section{Copyright and usage}

(c) The Royal College of Psychiatrists 2018
Selective serotonin reuptake inhibitors (SSRIs) such as sertraline, and cognitive-behavioural therapies such as prolonged exposure, are effective treatments for post-traumatic stress disorder (PTSD). ${ }^{1,2}$ Early response may provide an initial signal for a better prognosis in PTSD treatments as it is associated with better outcomes in psychotherapy ${ }^{3}$ and pharmacotherapy for depression, ${ }^{4}$ although definitions of early response vary in terms of timing, ${ }^{5-7}$ speed $^{8}$ and level ${ }^{3,5,6}$ of symptom reduction. For clinicians, understanding predictors of early response may inform resource allocation and treatment planning; ${ }^{9}$ and from a theoretical perspective, comparing early response between treatments may elucidate mechanisms of change. ${ }^{10}$ Therefore, identifying features of patients who respond early is critical. Pre-treatment characteristics (e.g. demographics and severity) are inconsistent predictors of early response, ${ }^{3,11}$ suggesting that alternative metrics warrant attention. More favourable attitudes about treatment are associated with early response to psychotherapy for $\mathrm{PTSD}^{12}$ and may explain some placebo effects in depression. ${ }^{13}$ Therefore, investigating expectancy of PTSD treatment outcome alongside early response is worthwhile.

Early response to prolonged exposure or sertraline for chronic PTSD was examined. Primary aims were to characterise early response across treatments and examine whether early response predicted better outcome and good end-state functioning, with good end-state functioning defined as non-clinical levels of PTSD, depression and anxiety. Our secondary aim, to help identify clinical markers of early response, was to examine whether pre-treatment symptom severity, demographic characteristics and treatment expectancy predicted early response. We hypothesised that early responders would be more likely to reach good end-state functioning, with gains maintained through follow-up, and that higher treatment expectancy would predict a greater likelihood of achieving early response.

\section{Method}

\section{Participants}

Men and women with PTSD participated in a multi-site randomised trial at University of Washington (Seattle, Washington) and Case Western Reserve University (Cleveland, Ohio) comparing prolonged exposure and sertraline. Of 200 patients who enrolled in the trial, the final sample was 134 patients who completed treatment, defined as completing seven or more prolonged exposure sessions (allowing for a minimally adequate dose of at least three sessions of exposure to the trauma memory) or at least 7 weeks on sertraline to achieve the target dose. Eligible participants had chronic DSM-IV-defined ${ }^{14}$ PTSD and were aged between 18 and 65 years.

Exclusion criteria, identified by clinical assessment, included current medically unstable bipolar disorder, depression requiring immediate admission to hospital or with psychotic features, severe self-injurious or suicidal attempt within the past 3 months, current schizophrenia or delusional disorder diagnosis, current alcohol or substance dependence diagnosis within the past 3 months, ongoing intimate relationship with perpetrator in cases of assault, unwillingness to discontinue trauma-focused psychotherapy or antidepressant medication (depending on treatment condition), failed trial of prolonged exposure (eight sessions or more) or sertraline $(150 \mathrm{mg} /$ day for at least 8 weeks) and medical contraindication for sertraline. For sample characteristics, see Table 1. Pretreatment characteristics did not differ between treatments or between treatment completers and the full intent-to-treat sample. 
Table 1 Pre-treatment patient characteristics by treatment condition

\begin{tabular}{|c|c|c|c|c|}
\hline \multirow{2}{*}{$\begin{array}{l}\text { Patient characteristics } \\
\text { Age (years) }\end{array}$} & \multicolumn{2}{|c|}{$\begin{array}{l}\text { Prolonged } \\
\text { exposure } \\
(n=77) \text {, mean } \\
\text { (s.d.) or \% }\end{array}$} & \multicolumn{2}{|c|}{$\begin{array}{l}\text { Sertraline } \\
(n=57), \text { mean } \\
\text { (s.d.) or \% }\end{array}$} \\
\hline & 36.61 & (11.55) & 39.72 & (12.01) \\
\hline Gender (\% female) & $83.1 \%$ & & $73.7 \%$ & \\
\hline Ethnicity (\% White) & $71.4 \%$ & & $71.9 \%$ & \\
\hline Time since trauma (years) & 12.23 & (12.24) & 12.58 & (13.67) \\
\hline \multicolumn{5}{|l|}{ Target trauma type } \\
\hline Adult sexual assault & $33.8 \%$ & & $31.6 \%$ & \\
\hline Childhood assault (e.g. CSA) & $24.7 \%$ & & $21.1 \%$ & \\
\hline Adult assault (non-sexual) & $19.5 \%$ & & $22.8 \%$ & \\
\hline Accident or natural disaster & $15.6 \%$ & & $15.8 \%$ & \\
\hline Death/violence to a loved one & $3.9 \%$ & & $7.0 \%$ & \\
\hline Combat/war & $2.6 \%$ & & $1.8 \%$ & \\
\hline PTSD severity (PSS-I) & 29.53 & (6.83) & 29.70 & (6.82) \\
\hline State of anxiety (STAI-S) & 55.29 & (11.36) & 57.03 & (11.73) \\
\hline Depression (BDI) & 24.09 & $(9.00)$ & 27.17 & $(9.89)$ \\
\hline $\begin{array}{l}\text { Expectancy of therapeutic outcome } \\
(\text { (ETO) })^{\mathrm{a}}\end{array}$ & 24.51 & (5.09) & 23.52 & (6.09) \\
\hline $\begin{array}{l}\text { a. } n=126 \text {. } \\
\text { BDI, Beck Depression Inventory; CSA, c } \\
\text { Therapeutic Outcome; PSS-I, PTSD Sym } \\
\text { stress disorder; STAl-S, State-Trait Anxi }\end{array}$ & - & $\begin{array}{l}\text { ault; E } \\
\text { ew; PT }\end{array}$ & post & $\begin{array}{l}\text { of } \\
\text { natic }\end{array}$ \\
\hline
\end{tabular}

\section{Treatments}

\section{Sertraline}

Ten weekly sessions (up to $30 \mathrm{~min}$, initial session of $45 \mathrm{~min}$ ) were provided by board-certified psychiatrists. The first session included the standard trauma interview and rationale for sertraline for PTSD, using a standardised manual and titration algorithm. ${ }^{15}$ Subsequent visits included general support and ongoing assessment of symptoms and medication side-effects. Dose was titrated from $25 \mathrm{mg} /$ day to a target dose of $200 \mathrm{mg} /$ day by week 5 . Dose was adjusted based on side-effect profile and clinical response, with options of reducing or maintaining previous dose. In this case, one more attempt was made to increase the dose, in line with standard clinical practice. The average final dosage across patients was $153.93 \mathrm{mg} /$ day (s.d. 57.04), with adherence monitored by pill counts and medication diaries. Medication for responders was provided through follow-up free of charge, if desired. For approximately $10 \%$ of the sample, an outside rater rated fidelity. Sertraline providers completed $96 \%$ of essential components with no protocol violations.

\section{Prolonged exposure}

Ten weekly sessions (90-120 $\mathrm{min}$ ) were delivered by masters- or $\mathrm{PhD}$-level clinicians trained in prolonged exposure. ${ }^{16}$ Components included psychoeducation, in vivo exposure (beginning after the second session), imaginal exposure (beginning in the third session), processing of the imaginal exposure and homework. For approximately $10 \%$ of the sample, an outside rater rated fidelity. Prolonged exposure providers completed $90 \%$ of essential components with no protocol violations. Therapist competence was rated very good (mean 2.73 , s.d. 0.32 ) on a scale from 1 (inadequate) to 3 (adequate or better).

\section{Measures}

\section{Interview measures}

The PTSD Symptom Scale - Interview (PSS-I) ${ }^{17}$ is a 17 -item semistructured interview assessing PTSD diagnosis and symptom severity. The PSS-I demonstrates good internal consistency, inter-rater reliability and test-retest reliability $(0.85,0.97$ and 0.80 , respectively). Over $10 \%$ of cases were independently re-rated, and inter- rater reliability was high for PTSD symptom severity (Intraclass Correlation Coefficient $=0.95)$ and diagnosis $(\kappa=1.00)$.

The Structured Clinical Interview for DSM-IV (SCID-IV) ${ }^{18}$ assessed inclusion and exclusion criteria. The SCID-IV has good inter-rater reliability. ${ }^{19}$ Over $10 \%$ of SCID-IVs were re-rated, and inter-rater reliability was acceptable $(\kappa=0.80)$.

\section{Self-report measures}

The PTSD Symptom Scale - Self-Report (PSS-SR) ${ }^{17}$ is a 17 -item measure of PTSD symptoms on a four-point scale from 0 (not at all) to 3 (five or more times per week/very much), with higher scores reflecting higher PTSD symptoms. The PSS-SR shows good internal consistency and test-retest reliability $(0.91$ and 0.74 , respectively). During acute treatment, this measure rated PTSD symptoms for the previous week and was used to identify early response.

The Beck Depression Inventory $(\mathrm{BDI})^{20}$ is a 21 -item measure of depressive symptoms for the past 2 weeks on a four-point scale, with higher scores reflecting greater depression. The BDI correlated well with interview measures of depression $(r=0.74)$ in psychiatric samples. $^{21}$

The Spielberger State-Trait Anxiety Inventory - State version (STAI-S) $)^{22}$ is a 20 -item measure of current anxiety symptoms for the past 2 weeks on a four-point scale from 1 (not at all) to 4 (very much so), with higher scores reflecting greater anxiety. The STAI-S has good test-retest reliability $(r=0.70)$.

The Expectancy of Therapeutic Outcome (ETO $)^{23}$ is a four-item measure (e.g. 'How successful do you think this treatment will be in reducing your trauma-related symptoms?') on a nine-point scale from 0 (not at all) to 8 (extremely), with higher scores reflecting higher treatment expectations and credibility. Although there are no specific psychometrics, this measure has been used in multiple clinical trials. ${ }^{23,24}$

\section{Early response definition}

Adapting the established definition used by Tadic et al, ${ }^{5}$ early response was defined as $20 \%$ or greater reduction in PTSD symptoms (PSS-SR) from pre-treatment to session 2. Calculating early response as a percentage change from pre-treatment severity rather than as a function of outcome makes it a candidate for being a pragmatic clinical indicator.

\section{Good end-state functioning definition}

Good end-state functioning was defined as experiencing subclinical symptoms across PTSD (PSS-I score $\leq 20$ ), depression (BDI score $\leq$ $10)$ and state of anxiety (STAI-S score $\leq 40)$. This captures a more comprehensive clinical picture than PTSD symptoms alone and is consistent with PTSD treatment literature ${ }^{25,26}$ Good end-state functioning was calculated at post-treatment and final follow-up.

\section{Procedure}

Patients were recruited by community advertising and clinical referrals. After potentially eligible patients reviewed study information and provided written informed consent, they completed interviews (using the PSS-I and SCID-IV) with independent evaluators blind to eventual treatment condition. Patients also completed self-report measures (PSS-SR, BDI and STAI-S), a physical examination, urine drug screen and pregnancy test, if female. Treatment was allocated by computer-generated urn randomisation sequence stratified by PTSD severity and current antidepressant use. Participants rated expectancy of therapeutic outcome (ETO) after the first session, with the provider being blind to the patients' responses, and completed self-report measure of PTSD symptom severity (PSS-SR) before each session. Independent raters conducted interview 
measures (e.g. PSS-I), and patients completed self-report measures (PSS-SR, BDI and STAI-S) at post-treatment and at 3, 6, 12 and 24 months' follow-up. On average, the last available follow-up assessment was at 21.21 months (s.d. 6.18). Patients were compensated for assessments. Ethical approvals were received from Institutional Review Boards at University of Washington (040541-D) and Case Western Reserve University/University Hospitals of Cleveland (08-03-47). This paper evaluates a combination of primary and secondary outcomes from a registered clinical trial that were assessed as planned, with no changes to follow-up periods or protocol (Clinicaltrials.gov identifier NCT00127673).

\section{Statistical analyses}

Analyses were conducted in SPSS version 22 for Windows. Predictors of early response were evaluated by chi-squared analyses and logistic regressions. Moderators were explored with SPSS regression path analysis modelling tools ModProbe and PROCESS, ${ }^{21}$ which are widely used in health sciences for estimating direct and indirect effects, and interactions.

\section{Results}

\section{Characterising early response}

The overwhelming majority of patients achieved at least $20 \%$ reduction from pre-treatment PTSD symptoms (PSS-SR) at some time during treatment with sertraline $(89.5 \%)$ and prolonged exposure (92.2\%). As shown in Fig. 1, 20\% reduction in symptoms was most commonly met by the second session in both sertraline $(42.1 \%)$ and prolonged exposure (40.3\%).

\section{Early response predicts good end-state functioning Post-treatment good end-state functioning}

As illustrated in Fig. 2, using moderation analysis in ModProbe ${ }^{27}$ to examine the relationship among treatment modality, early response and good end-state functioning, there was a significant interaction between treatment type and early response $(B=2.18, t=7.19, P=$ $0.01)$, such that early response predicted good end-state functioning at post-treatment in sertraline only $(B=2.55, t=14.88, P<0.001$, $95 \%$ CI 1.26-3.85). For those receiving sertraline, the proportion achieving good end-state functioning at post-treatment was higher for early responders (78.3\%) compared with non-early responders (21.9\%; Number Needed to Treat $=1.8$, 95\% CI 1.28 3.00). Of note, there was no difference in final sertraline dose (in

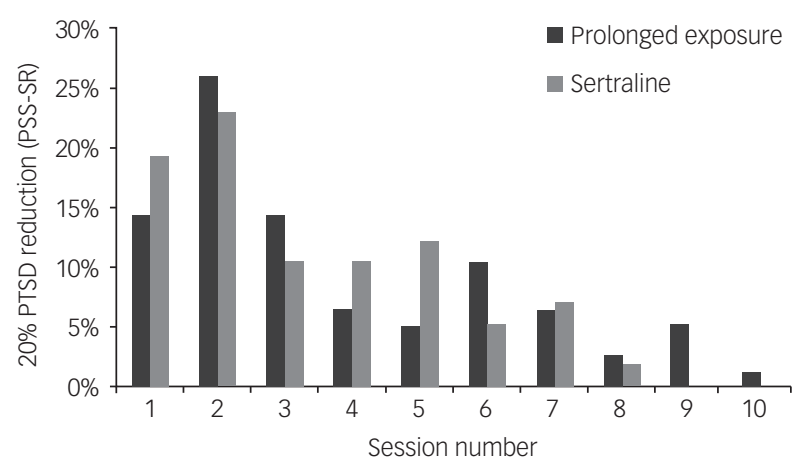

Fig. 1 Session at which PTSD symptom severity (PSS-SR) reduces by $20 \%$. Session refers to first occurrence of $20 \%$ symptom reduction from pre-treatment. PSS-SR, PTSD Symptom Scale - SelfReport; PTSD, post-traumatic stress disorder.

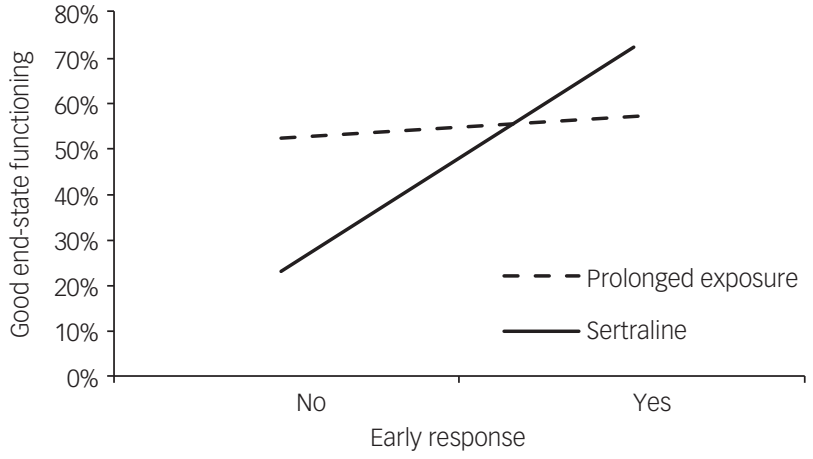

Fig. 2 Early response predicts good end-state functioning in sertraline only. Early response was defined as $20 \%$ reduction in PTSD symptoms from baseline by session 2. Good end-state functioning was defined by sub-clinical symptoms across PTSD (PSS-I $\leq 20)$, depression (BDI $\leq 10)$ and state of anxiety (STAI-S $\leq 40$ ). For prolonged exposure, $B=0.37, t=0.62, P$ (not significant), $95 \% \mathrm{Cl}$ -0.55 to 1.30 ; for sertraline, $B=2.55, t=14.88, P<0.001,95 \% \mathrm{Cl}$ 1.26-3.85. BDI, Beck Depression Inventory; PSS-I, PTSD Symptom Scale - Interview; PTSD, post-traumatic stress disorder; STAI-S, State-Trait Anxiety Inventory - State.

milligrams) between early responders (mean 155.71, s.d. 60.84) and non-early responders (mean 152.64, s.d. 55.04), or between those who achieved good end-state functioning (mean 147.00, s.d. 65.07 ) and those who did not (mean 156.67 , s.d. 50.83). In prolonged exposure, the likelihood of achieving good end-state functioning did not differ for early responders compared with nonearly responders.

\section{Long-term follow-up good end-state functioning}

Early response only predicted good end-state functioning at final follow-up in sertraline $(B=1.39, t=4.86, P=0.03$, 95\% CI 0.15 2.62 ), but not in prolonged exposure (values were not significant). Similar to post-treatment, for those receiving sertraline, the likelihood of achieving good end-state functioning at final follow-up was higher for those who responded early $(72.7 \%)$ compared with those who did not $(40.0 \%$; Number Needed to Treat $=3.1,95 \%$ CI $1.68-16.71)$. Again, sertraline dose at final follow-up did not differ between those who achieved good end-state functioning at final follow-up and those who did not, and early response did not predict good endstate functioning at follow-up in prolonged exposure.

\section{Predictors of early response}

Possible pre-treatment predictors of early response were evaluated, including gender (male, 0 ; female, 1), trauma type (adult sexual assault, adult assault (non-sexual), child physical or sexual assault, accident or natural disaster, death/violence to a loved one or combat/war), age, time since trauma, pre-treatment state of anxiety (STAI-S), depression severity (BDI), PTSD severity (PSSI) and expectancy of therapeutic outcome. Only two variables were significantly associated with early response: higher expectancy of positive treatment outcome $(\beta=0.12, t(1)=6.94, P=0.008,95 \%$ CI 1.03-1.22) and lower PTSD severity (PSS-I) $(\beta=-0.12, t(1)=$ 9.32, $P=0.002$, 95\% CI $0.83-0.96$ ).

To examine whether these effects were moderated by treatment type, we used moderation analyses in PROCESS. ${ }^{28}$ As shown in Fig. 3, higher pre-treatment PTSD severity predicted moderately lower likelihood of early response in prolonged exposure $(B=$ $-1.11, P<0.001,95 \%$ CI -1.72 to -0.49$)$ but not in sertraline (values were not significant). Conversely, Fig. 3 also shows that 

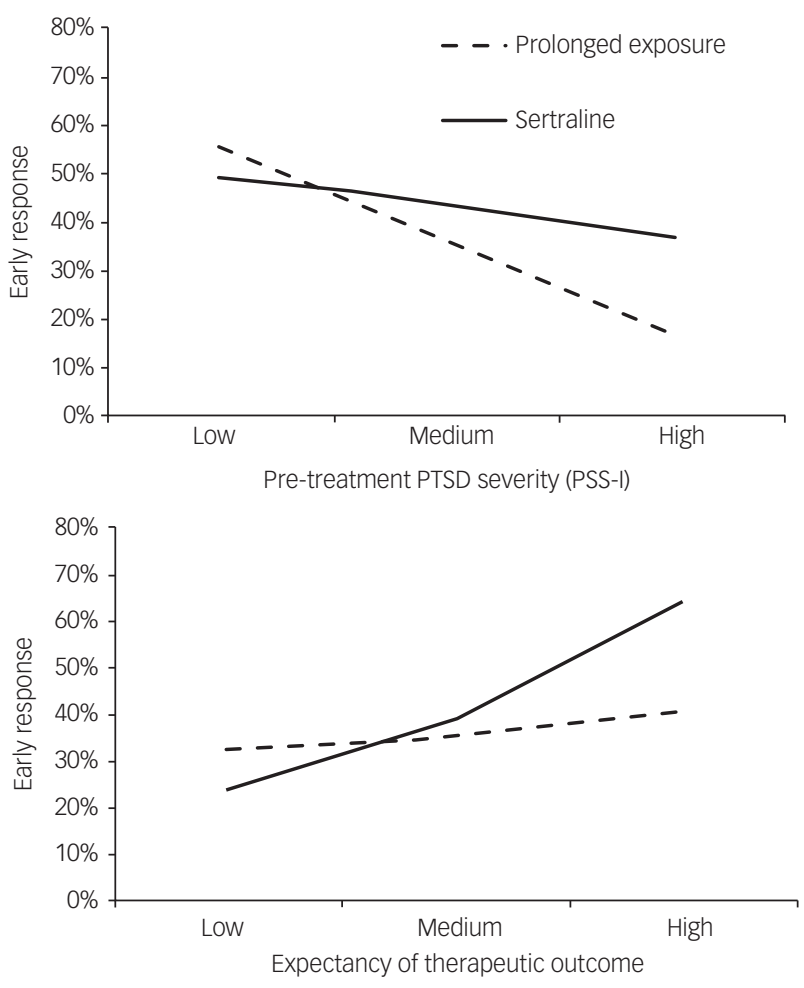

Fig. 3 Pre-treatment PTSD severity and expectancy predicting early response by treatment condition. Higher pre-treatment PTSD severity predicted early response in prolonged exposure but not sertraline. Higher expectancy predicted early response in sertraline but not prolonged exposure. Values represent estimated likelihood of early response for patients with low (25th percentile), medium (50th percentile) and high (75th percentile) pre-treatment PTSD severity or expectancy, separated by treatment type. For pretreatment PTSD severity: in prolonged exposure, $B=-1.11, P<$ $0.001,95 \% \mathrm{Cl}-1.72$ to -0.49 ; in sertraline, $B=-0.31, P$ (not significant), $95 \% \mathrm{Cl}-0.88$ to -0.26 . For expectancy of therapeutic outcome: in prolonged exposure, $B=0.26, P$ (not significant), $95 \% \mathrm{C}$ -0.29 to 0.81 ; in sertraline, $B=1.35, P=0.01,95 \% \mathrm{Cl} 0.35-2.36$. PSS I, PTSD Symptom Scale - Interview; PTSD, post-traumatic stress disorder.

higher expectancy of therapeutic outcome predicted higher likelihood of early response in sertraline ( $B=1.35, P=0.01,95 \%$ CI $0.35-2.36)$ but in not prolonged exposure (values were not significant).

Given that higher expectancy predicted a greater likelihood to achieve early response in sertraline, and early response predicted greater likelihood of good end-state functioning at post-treatment, we used PROCESS ${ }^{28}$ to examine whether the relationship between expectancy and good end-state was mediated by early response in sertraline. Indeed, the relationship between higher expectancy ratings and higher likelihood of achieving good end-state functioning was mediated by early response in sertraline $(P=0.004,95 \% \mathrm{CI}$ $0.68-3.68)$, thus arguing that patients' expectancies about sertraline being helpful may influence good end-state functioning by driving early response.

\section{Discussion}

Patients with chronic PTSD who responded early to sertraline were approximately four times more likely to achieve and maintain nonclinical levels of PTSD, depression and anxiety. Further, this study indicates that higher expectancy of better treatment outcome at the very first session, even before the first pill has been taken, may set the stage for a later cascade of neurobiological effects and behavioural changes, being associated with early symptom reduction that was further associated with better outcomes through long-term follow-up. Despite similar rates of early response in prolonged exposure and sertraline, the beneficial effect of early response was observed in sertraline only.

Early response to sertraline was strongly associated with higher odds of achieving and maintaining good end-state functioning. Consensus is lacking regarding whether early response to pharmacotherapy for depression and anxiety disorders reflects true psychotropic $^{4,29}$ or placebo effects ${ }^{30}$ and regarding specific mechanisms through which placebo effects occur. ${ }^{31}$ Although the current study did not include a placebo comparison to disentangle pharmacological effects from placebo effects, the findings suggest that early PTSD response to sertraline is an important clinical signal for later prognosis. Pre-treatment severity or patient demographic characteristics did not reliably predict early response in sertraline, arguing against the utility of these indices to early response; however, patients with high expectations of improving from sertraline were more likely to achieve early response than those with lower expectations. Importantly, early response explained some of the association between expectancy and outcome, suggesting early response may be a pathway by which expectancies enhance likelihood of achieving strong positive outcomes. However, it is possible that early responders with higher expectancies were likely to be less severe and show more emotional responsiveness, and thus respond better to sertraline. Consistent with expectancy mediating placebo response in depression, ${ }^{32}$ pre-treatment expectancy of potential helpfulness may play a meaningful role in its long-term effectiveness for PTSD alongside active serotonergic effects.

One plausible explanation is a cascade-like effect, such that higher expectancy of sertraline coupled with early response creates optimal conditions for overall symptom reduction. It may be that immediate confirmation of positive expectancy in the form of early symptom reduction propels good outcomes, rather than expectancy in isolation, and may trigger neurobiological processes to induce physiological change. ${ }^{31}$ Belief that treatment will work may lead to improvements in mood and mood regulation, or increase behavioural activation. Although expectancy may also influence psychotherapy processes, treatment beliefs may be less critical in prolonged exposure where expectancy asserts a less powerful effect, such that even sceptical clients are likely to improve over time. The specific pathway between expectancy and early response is not clear. Clinically, treatment expectations can easily be assessed via a few brief questions after the first session after the therapeutic rationale has been presented, and session-by-session PTSD severity can be easily monitored through a brief self-report questionnaire, and therefore feasibly included into routine clinical practice. However, it should be noted that although early response in sertraline is an important signal of overall better prognosis, the absence of early response in sertraline does not necessarily justify changing the course of treatment as symptom reduction occurred in later sessions for the vast majority of patients receiving sertraline (see also Foa et $\mathrm{l}^{26}$ ).

Although rates of early response were similar in prolonged exposure and sertraline, the association with better outcome was seen in sertraline only. This is in contrast to a comparison of cognitive-behavioural therapy and sertraline for depression, where this effect was observed in both treatments. ${ }^{5}$ It is possible that the overall higher rates of remission in prolonged exposure for PTSD ${ }^{33}$ mean that early and later responders alike are able to benefit from treatment. Alternatively, the exposure and processing components of prolonged exposure that theoretically underpin therapeutic change ${ }^{26}$ are introduced after the second session; and it may be that patients' expectations for change are more concretely linked to the start of these components. Patients with more severe PTSD in prolonged exposure, but not sertraline, were less likely to 
experience early response. Clinically, similar to sertraline and perhaps more so, a lack of early response is not a marker for later treatment failure and should not be interpreted as a cause for altering prolonged exposure treatment.

In this study, patients received key components of prolonged exposure and followed a standard titration algorithm for sertraline to systematically examine the role of early response on later treatment outcome. Generalisation to less structured interventions was not evaluated, nor were alternative effective psychotherapies for PTSD. ${ }^{2}$ There was also no placebo control; nevertheless, the therapeutic effect of sertraline and prolonged exposure above waitlist and placebo is well established, ${ }^{1}$ arguing against expectations or time solely accounting for observed effects.

Responding early to sertraline for PTSD meaningfully increased patients' likelihood of recovery. This effect was particularly strong when patients held higher expectations that sertraline would work for them. As such, clinicians ought to assess expectancy before starting sertraline and be cognisant of a potential beneficial cascade effect between expectancy and early response when treating patients with sertraline for PTSD. For patients with lower expectancy, early and repeated discussions about why and how SSRIs work and carefully addressing patients' concerns may be particularly important. Future research should further examine the conditions that fuel higher expectancy and early symptom reduction for those receiving SSRIs for PTSD, as they offer an important combined signal of recovery.

Belinda Graham, DClinPsy (D), Postdoctoral Fellow, Department of Psychology, University of Washington, USA; Natalia M. Garcia, MS, Graduate Student, Department of Psychology, University of Washington, USA; Mark S. Burton, MA, Graduate Student, Department of Psychological Sciences, Case Western Reserve University, USA; Andrew A. Cooper, PhD, Postdoctoral Fellow, Department of Psychological Sciences, Case Western Reserve University, USA; Peter P. Roy-Byrne, MD, Professor, Department of Psychiatry and Behavioral Sciences, Harborview Medical Center, USA: Matig R. Mavissakalian MD, Professor, Case Western Reserve University School of Medicine, University Hospitals Cleveland Medical Center, USA; Norah C. Feeny, PhD, Professor, Department of Psychological Sciences, Case Western Reserve University, Professor, Department of Psychological Sciences, Case Western Reserve Univers
USA; Lori A. Zoellner, PhD, Professor, Department of Psychology, University of Washington, USA

Correspondence: Belinda Graham, Department of Experimental Psychology, University of Oxford, The Old Rectory, Paradise Square, Oxford OX1 1TW, UK. Email: belinda.graham@psy.ox.ac.uk

First received 4 May 2018, final revision 24 Aug 2018, accepted 7 Sep 2018

\section{Acknowledgements}

The authors would like to thank University of Washington's Helen R. Whiteley Center at Friday Harbor Laboratories for the use of their facilities during the initial conceptualisation of this paper.

\section{References}

1 Watts BV, Schnurr PP, Mayo L, Young-Xu Y, Weeks WB, Friedman MJ. Metaanalysis of the efficacy of treatments for posttraumatic stress disorder. J Clin Psychiatry 2013; 74(6): e541-50

2 Cusack K, Jonas DE, Forneris CA, Wines C, Sonis J, Middleton JC, et al. Psychological treatments for adults with posttraumatic stress disorder: a systematic review and meta-analysis. Clin Psychol Rev 2016; 43: 128-41.

3 Lewis CC, Simons AD, Kim HK. The role of early symptom trajectories and pretreatment variables in predicting treatment response to cognitive behavioral therapy. J Consult Clin Psychol 2012; 80(4): 525-34.

4 Szegedi A, Jansen WT, van Willigenburg AP, van der Meulen E, Stassen $H$, Thase ME. Early improvement in the first 2 weeks as a predictor of treatment outcome in patients with major depressive disorder: a meta-analysis including 6562 patients. J Clin Psychiatry 2009; 70(3): 344-53.

5 Tadić A, Helmreich I, Mergl R, Hautzinger M, Kohnen R, Henkel V, et al. Early improvement is a predictor of treatment outcome in patients with mild major, minor or subsyndromal depression. J Affect Disord 2010; 120(1-3): 86-93.

6 Taylor MJ, Freemantle N, Bhagwagar Z, Geddes JR. Early onset of selective serotonin reuptake inhibitor antidepressant action: systematic review and meta-analysis. Arch Gen Psychiatry 2006; 63(11): 1217-23.
7 Hayes AM, Feldman GC, Beevers CG, Laurenceau J-P, Cardaciotto L, LewisSmith J. Discontinuities and cognitive changes in an exposure-based cognitive therapy for depression. J Consult Clin Psychol 2007; 75(3): 409-21.

8 Haas E, Hill RD, Lambert MJ, Morrell B. Do early responders to psychotherapy maintain treatment gains? J Clin Psychol 2002; 58(9): 1157-72.

9 Whipple JL, Lambert MJ, Vermeersch DA, Smart DW, Nielsen SL, Hawkins EJ. Improving the effects of psychotherapy: the use of early identification of treatment and problem-solving strategies in routine practice. J Couns Psychol 2003; 50(1): 59-68.

10 Tang TZ, DeRubeis RJ. Reconsidering rapid early response in cognitive behavioral therapy for depression. Clin Psychol Sci Pract 1999; 6(3): 283-8.

11 Nordberg SS, Castonguay LG, Fisher AJ, Boswell JF, Kraus D. Validating the rapid responder construct within a Practice Research Network. J Clin Psychol 2014; 70(9): 886-903

12 Westra HA AJ, Marcus M. Expectancy, homework compliance, and initial change in cognitive-behavioral therapy for anxiety. J Consult Clin Psychol 2007; 75(3): 363-73.

13 Snyder C, Ingram RE. Handbook of Psychological Change: Psychotherapy Processes \& Practices for the 21st Century. vol. xvi. John Wiley \& Sons, 2000.

14 American Psychiatric Association. Diagnostic and Statistical Manual of Mental Disorders (4th ed., Text Revision). APA, 2000.

15 Marshall RD, Beebe KL, Oldham M, Zaninelli R. Efficacy and safety of paroxetine treatment for chronic PTSD: a fixed-dose, placebo-controlled study. Am J Psychiatry 2001; 158(12): 1982-8.

16 Foa E, Hembree E, Rothbaum BO. Prolonged Exposure Therapy for PTSD: Emotional Processing of Traumatic Experiences (Treatments That Work). Oxford University Press, 2007.

17 Foa EB, Riggs DS, Dancu CV, Rothbaum BO. Reliability and validity of a brief instrument for assessing post-traumatic stress disorder. J Trauma Stress 1993; 6(4): 459-73.

18 First M, Spitzer R, Gibbon M, Williams J. Structured Clinical Interview for DSM-IV. New York State Psychiatric Institute, 1995.

19 Lobbestael J, Leurgans M, Arntz A. Inter-rater reliability of the Structured Clinical Interview for DSM-IV Axis I Disorders (SCID I) and Axis II Disorders (SCID II). Clin Psychol Psychother 2011; 18(1): 75-9.

20 Beck AT, Ward C, Mendelsohn M. Beck Depression Inventory (BDI). Arch Gen Psychiatry 1961; 4(6): 561-71.

21 Beck AT, Epstein N, Brown G, Steer RA. An inventory for measuring clinical anxiety: psychometric properties. J Consult Clin Psychol 1988; 56(6): 893-7.

22 Spielberger CD, Gorsuch RL, Lushene PR, Vagg PR, Jacobs AG. Manual for the State-Trait Anxiety Inventory (Form Y). Consulting Psychologists Press, 1983.

23 Foa EB, Rothbaum BO, Riggs DS, Murdock TB. Treatment of posttraumatic stress disorder in rape victims: a comparison between cognitive-behavioral procedures and counseling. J Consult Clin Psychol 1991; 59(5): 715-23.

24 Resick PA, Nishith P, Weaver TL, Astin MC, Feuer CA. A comparison of cognitive-processing therapy with prolonged exposure and a waiting condition for the treatment of chronic posttraumatic stress disorder in female rape victims. J Consult Clin Psychol 2002; 70(4): 867-79.

25 Feeny NC, Zoellner LA, Mavissakalian MR, Roy-Byrne PP. What would you choose? Sertraline or prolonged exposure in community and PTSD treatment seeking women. Depress Anxiety 2009; 26(8): 724-31.

26 Foa EB, Dancu CV, Hembree EA, Jaycox LH, Meadows EA, Street GP. A comparison of exposure therapy, stress inoculation training, and their combination for reducing posttraumatic stress disorder in female assault victims. J Consult Clin Psychol 1999; 67(2): 194-200.

27 Hayes AF, Matthes J. Computational procedures for probing interactions in OLS and logistic regression: SPSS and SAS implementations. Behav Res Methods 2009; 41(3): 924-36.

28 Hayes AF. Introduction to Mediation, Moderation, and Conditional Process Analysis: A Regression-Based Approach. Guilford Press, 2013.

29 Machado-Vieira R, Salvadore G, Luckenbaugh DA, Manji HK, Zarate CA. Rapid onset of antidepressant action: a new paradigm in the research and treatment of major depression. J Clin Psychiatry 2008; 69(6): 946-58.

30 Quitkin FM, Rabkin JG, Ross D, McGrath PJ. Duration of antidepressant drug treatment: what is an adequate trial? Arch Gen Psychiatry 1984; 41(3): 238-45.

31 Peciña M, Zubieta J-K. Molecular mechanisms of placebo responses in humans. Mol Psychiatry 2015; 20(4): 416-23.

32 Rutherford BR, Wall MM, Brown PJ, Choo T-H, Wager TD, Peterson BS, et al. Patient expectancy as a mediator of placebo effects in antidepressant clinical trials. Am J Psychiatry 2017; 174(2): 135-42.

33 Powers MB, Halpern JM, Ferenschak MP, Gillihan SJ, Foa EB. A meta-analytic review of prolonged exposure for posttraumatic stress disorder. Clin Psychol Rev 2010; 30(6): 635-41. 